\title{
Editorial. Les réseaux internationaux de violence
}

\section{Daniel Hermant}

\section{(2) OpenEdition}

Journals

Édition électronique

URL : http://journals.openedition.org/conflits/748

DOI : $10.4000 /$ conflits.748

ISSN : $1777-5345$

Éditeur :

CCLS - Centre d'études sur les conflits lilberté et sécurité, L'Harmattan

Édition imprimée

Date de publication : 17 janvier 1991

ISSN : 1157-996X

Référence électronique

Daniel Hermant, «Editorial. Les réseaux internationaux de violence », Cultures \& Conflits [En ligne], 04 | hiver 1991, mis en ligne le 07 janvier 2003, consulté le 30 mars 2021. URL : http://

journals.openedition.org/conflits/748; DOI : https://doi.org/10.4000/conflits.748

Ce document a été généré automatiquement le 30 mars 2021.

Creative Commons License 


\title{
Editorial. Les réseaux internationaux de violence
}

\author{
Daniel Hermant
}

Réseaux internationaux de violence, l'expression peut paraitre obscure mais elle est la seule à rendre compte de la violence des attentats dits terroristes, de celle engendrée par des trafics aussi divers que celui de la drogue, des explosifs ou des armements lourds, ainsi que de l'activité des administrations qui parfois les animent ou de la politique de certains états. Le champ ainsi balisé concerne autant les relations interétatiques que celles existant entre états, guérillas, réseaux mafieux ou organisations clandestines c'est-à-dire entre "acteurs souverains" et acteurs "libres de souveraineté" pour reprendre le vocabulaire de J. N. Rosenau. On aura donc à analyser dans ce numéro une dynamique en réseau caractérisée par la nature des "transferts de violence" et structurée au niveau formel par les relations "horizontales" liant entre eux les états, ou par celles "obliques" existant entre états acheteurs d'armes, trafiquants II y a là un champ particulier souvent mal perçu par les analystes qui en méconnaissent la complexité. En effet la connaissance de ces réseaux internationaux de violence reposait jusqu'à présent soit sur une littérature ponctuelle, journalistique, qui s'intéressait surtout à la dimension oblique des multiples trafics qu'ils soient d'état ou non :

Irangate, Contragate, affaire Luchaire, route des Balkans, fourniture d'armes à la Croatie par les milices libanaises mais qui était incapable de l'insérer dans une analyse globale de relations internationales, soit sur une littérature universitaire qui, si elle savait replacer la question des transferts d'armes dans les politiques d'état et les relations internationales, négligeait de les lier à l'activité directe des acteurs subétatiques. Les contributions que nous réunissons cherchent, dans l'esprit des débats d'une des tables rondes du congrès de l'Association Internationale de Science Politique qui s'est tenue à Buenos Aires en juillet dernier'1 à mêler les deux approches. Elles ouvrent des perspectives nouvelles et, espérons-le, fécondes. Pour mettre en évidence cette dynamique en réseau, on peut bâtir une "anatomie" à partir de l'examen de l'activité des acteurs et de leurs emboîtements. Le premier réseau isolable est celui "horizontal" des relations d'état à état. Ces relations ont la visibilité administrative, et quelquefois médiatique, du commerce des armes, activité parfaitement légale dans 
laquelle les gouvernements jouent un rôle crucial en produisant et en exportant euxmêmes les armes au travers de sociétés publiques, ou en en contrôlant l'exportation par des sociétés privées soumises à des autorisations administratives. La nature officielle de ces activités fait qu'elles se déroulent souvent dans le cadre d'accords bilatéraux d'état à état. Simple chaînon technique dans des transactions entre gouvernements, ces opérations commerciales en définitive classiques, se prêtent à une analyse en terme de flux de marchandises. On peut donc établir la géographie des producteurs d'armes, les caractériser selon l'éventail de leur catalogue, mesurer leur dynamisme de vendeur, déterminer leurs clients. Mais les difficultés techniques propres au décryptage des documents administratifs limitent en général l'analyse à ce niveau d'explication. Les auteurs mettent en évidence la nature largement étatisée de ce commerce, nul n'en sera surpris; ils dessinent également le cercle fermé des producteurs de systèmes majeurs d'armement et en montrent la solidité : malgré la vive pression de certains pays : Chine, Argentine, Brésil, Israël, la concentration de la production de pointe reste la règle. Le second type d'emboîtement articule justement états, administrations, mouvements de guérilla ou organisations clandestines. Il s'agit de relations obliques, dissymétriques dont, contrairement au cas précédent, la visibilité est partielle. Gouvernements, bureaucraties, sociétés privées d'armements, réseaux de trafiquants, acteurs mafieux, organisations clandestines ou mouvements de lutte armée ont une logique et des intérêts propres : aussi la mise en regard des deux bouts de la chaîne n'éclaire-t-elle que modérément celle-ci. Pour le dire autrement le maillon central du réseau possède une autonomie forte par rapport à ses tenants et aboutissants, mais celle-ci n'est perceptible qu'à condition de se départir du paradigme réaliste et étatiste en décalant l'analyse pour la focaliser sur les acteurs transnationaux subétatiques considérés comme des acteurs directement politiques et ce, même à l'échelon international. La configuration la plus fréquente nous est fournie par les relations guérilla/état où le transfert d'armes a un effet direct sur la production de la violence. Le besoin d'armes de la première, la volonté politique du second se rencontrent non pas directement, mais par l'intermédiaire d'un réseau forgé dans les couloirs des bureaucraties de l'état fournisseur. Pourtant ce chaînon n'est pas homogène et les individus qui l'animent poursuivent parfois des objectifs contraires à ceux du gouvernement comme le montrent les mécanismes de l'Irangate ou de l'affaire Luchaire. Si l'action de ces bureaucraties peut être fonctionnelle dans le cadre d'une politique étrangère secrète, dans un autre contexte elle s'autonomise jusqu'à gérer directement les exportations d'armement comme le dit notamment R. Bates Gill à propos de la Chine, et comme on peut le supposer pour l'ex-URSS. Ces bureaucraties sont d'autant plus tentées d'utiliser des réseaux de trafiquants qu'elles suivent une politique en contradiction avec celle du gouvernement. Dès lors des transactions, voire des collusions, se produisent entre membres de l'appareil gouvernemental des services secrets ou de renseignement et responsables de sociétés industrielles ou d'importexport au profil ambigu. Ces trafiquants, partie prenante d'un marché plus vaste, sont polyvalents. Ils jouent de leurs activités et de leurs contacts légaux pour devenir des intermédiaires "obligés" lorsque le producteur primaire ne veut pas connaître le destinataire. Les trafiquants opérant sur le marché des armes peuvent même imposer des conditions léonines obligeant de petites organisations à "enlever" et à $\mathrm{K}$ "payer" un package de matériel dont elles n'ont pas vraiment usage. En position parasitaire, ils peuvent en fonction des circonstances se retourner contre le fournisseur ou contre le destinataire. Leur faiblesse provient essentiellement de leur taille, aussi se contentent- 
ils en général de "coups" et n'assurent-ils pas, à la différence des bureaucraties officielles, un approvisionnement régulier des guérillas.

Ce cas de figure a de fortes similitudes avec les pratiques d'un certain nombre d'états dits du tiers-monde soucieux de compléter leur arsenal classique par l'acquisition d'armes beaucoup plus "déstabilisantes". Pour atteindre cet objectif, ces gouvernements utilisent la corruption individuelle, le racket discret d'entreprises ou encore pratiquent le détournement systématique de technologies civiles à des fins militaires, c'est-à-dire se positionnent dans l'espace clandestin des affaires d'espionnage industriel ou de la "subversion" comme disent les services de renseignements. Les tentatives de divers pays - et notamment de l'Irak - pour obtenir malgré les interdictions, l'arme nucléaire, illustrent ce cas de figure où seule l'investigation policière peut faire apparaître le lien entre les agents de l'état étranger et leurs contacts. Seule différence, mais de taille, avec la configuration que nous envisagions plus haut, il n'y a pas ici contradiction, mais subordination directe entre la volonté politique de l'état initiateur du réseau et les pratiques de sa bureaucratie : c'est l'acheteur qui contrôle et non le vendeur. Les réseaux dits "terroristes" dont l'activité ne porte pas sur des transferts massifs d'armement et, par là même, n'induit pas une circulation d'argent comparable à celle des cas précédents constituent un troisième type d'emboîtement qu'on pourrait qualifier de mixte parce qu'il reprend des caractéristiques appartenant aux deux configurations que nous avons déjà isolées. D'une certaine manière ce sont des réseaux de prestations de services. Ils reposent sur la circulation ponctuelle d'une volonté politique, et d'un savoir-faire. Ces réseaux contrairement aux précédents sont beaucoup plus intégrés. Le maillon central se réduit à un transfert discret de marchandises (explosifs), ou au déplacement de quelques individus susceptibles de commettre des attentats. Bien qu'il n'y ait pas un modèle général, mais une collection de cas particuliers, on peut dire que ces réseaux transnationaux de violence reposent sur l'homogénéité de leurs composants, voire sur leur "intégration": il n'y a pas de solution de continuité entre l'organisation qui effectue les attentats et celle externe qui commandite, mais simplement une inévitable asymétrie entre petites équipes souvent constituées d'une manière empirique. Comme il se doit la visibilité de ces réseaux est faible ou nulle. Clandestins par nécessité ils n'apparaissent dans l'actualité que lorsqu'ils effectuent des attentats. On les connaît quand ils sont démantelés : lors de procès par exemple, ou encore quand des évolutions politiques rendent publiques des informations jusque-là confidentielles. Ce fut récemment le cas avec l'implosion du monde communiste qui jeta (presque) les dossiers $\mathrm{du}$ "réseau de la terreur" sur la place publique ${ }^{2}$. Cette documentation difficile à manier, mais d'ores et déjà disponible pour le chercheur patient, renouvelle la connaissance du dossier et permet notamment d'éclairer d'un jour nouveau la question controversée de l'appui des états aux organisations clandestines. La dissection du réseau Ali Fouad Saleh que nous présentons ci-après met ainsi en évidence l'interface organisation clandestine/groupe communautaire/état et dessine l'emboîtement subtil de ces acteurs ${ }^{3}$. On peut conclure de cet examen anatomique que le maillon central du réseau se comporte rarement comme un interface neutre, technique, uniquement animé de l'extérieur, mais qu'il constitue la plupart du temps un véritable milieu ayant une logique financière, politique ou mafieuse. Plus généralement les réseaux ne renvoient pas à un formalisme créé par l'analyste mais à un ensemble qui trouve son sens dans les objectifs des acteurs situés en amont ou en aval. Ce sont eux qui non seulement amorcent et assurent la continuité de la chaîne relationnelle, mais qui l'insèrent dans 
les relations internationales la séparant ainsi d'autres réseaux comme ceux du grand banditisme dont les activités restent en général criminelles ${ }^{4}$. En focalisant l'analyse sur cette dimension il est possible d'interpréter les effets des réseaux, comme ce numéro en a l'ambition, sans tomber dans l'anecdote ou le sensationnel.

$C^{\prime}$ 'est en effet à travers les contradictions de la politique des états-vendeurs déchirés entre le profit commercial et la volonté géopolitique; mais également à travers celle des états-acheteurs pour qui la dimension économique, bien que secondaire, se heurte souvent à l'impératif stratégique, que les contributions que nous réunissons dans ce numéro décrivent le champ doublement polarisé où s'inscrivent les effets des réseaux de transferts d'armement. La première polarité de cet espace de contradictions est donc économique. Le poids industriel et financier des ventes d'armes pour les pays producteurs, la croyance dans l'effet de développement induit par les technologies militaires, sans parler d'un réflexe de "thésaurisation militaire", très fréquent dans le tiers-monde, sous-tendent ce qu'on pourrait qualifier "d'économie politique" du marché des armes. Le commerce des armes évolue en fonction d'une conjoncture économique dont nos auteurs esquissent les dernières inflexions. Celles-ci, marquées par la crise économique, par la diminution des capacités de paiement de nombre de clients, par le développement des pratiques de "retrofiting" au détriment du renouvellement des matériels entraînent la morosité : comment va-t-on financer les prochains programmes d'armements si on ne parvient plus à diminuer les coûts de fabrication par l'allongement des séries que rendait possible l'exportation? Ou plus abruptement exprimé : la France n'a vendu aucun Mirage 2000 à l'exportation l'année dernière. La deuxième polarité est politique ou même géopolitique. Fournir des armes c'est projeter son influence à l'extérieur, se constituer une clientèle : un changement de fournisseur est souvent le signe d'un changement de camp, rappelons-nous l'égypte de Nasser. Par des ventes, ou par une aide appropriée, un état peut en stabiliser un autre, dissuader un voisin belliqueux d'intervenir, contribuer au calme d'une zone; inversement il peut aggraver la violence régionale, et prolonger un conflit. Ces effets résultent de mécanismes complexes qu'illustrent la guerre irano-irakienne analysée par K Krause: il s'agissait d'empêcher une déstabilisation régionale, dont les conséquences étaient imaginées dans toutes les capitales occidentales comme catastrophiques, par des transferts (on n'ose pas dire ventes) d'armements de la France et de l'URSS vers l'Irak. En regard l'Iran, pays agressé, mais devenu agresseur parce qu'il voulait "à tout prix" faire tomber le "Nabuchodonosor de Bagdad", était soumis à un embargo occidental, secrètement atténué pour cause de tractations sur les otages, mais bénéficiait de l'obligeance intéressée des Israéliens et de l'apport chinois dont $\mathrm{R}$. Bates Gill nous dit toute la complexité. Ainsi l'équilibre des belligérants provient bien souvent de ce que K. Krause appelle le "pouvoir structurel" des vendeurs d'armes.

Mais cette géopolitique peut être lue à l'envers. Les états cherchent à gêner leurs adversaires en alimentant des guérillas, ce qui est encore de "bonne guerre" et s'inscrit dans une logique de "camp". Il peut aussi s'agir, si on prend le point de vue des acheteurs, de se renforcer face à ses voisins en accumulant un arsenal, signe et réalité d'une volonté d'expansion régionale, ce qui s'inscrit encore dans un cadre global (puissance relais) mais peut également en sortir (Iran avant ou après 79). Enfin on peut aussi se renforcer face aux plus grands en acquérant par exemple ces armes de statut que sont les armes nucléaires, on contrevient alors à la hiérarchie reconnue des puissances comme le remarque F. S. Pearson. Le cas des "réseaux légers de terrorisme" est particulier, ils visent à se venger, mais également quand ils sont animés par des 
états, à rééquilibrer des rapports de force très inégaux ce qui les replace dans le cadre de cette géopolitique contestataire que nous évoquions à l'instant. C'est en tenant compte de cette situation complexe mais également en n'oubliant pas l'émiettement des centres de décision ou de la volonté étatique dont nous avons esquissé le mécanisme plus haut en analysant rapidement les rapports entre bureaucraties et trafiquants, qu'une typologie des réseaux de violence peut être esquissée. Elle permet de distinguer : des réseaux déstabilisateurs caractérisés par des transferts immédiats, directs, sans inertie, assez bien contrôlés par les acteurs qui les mettent en oeuvre (réseaux dit terroristes). Des réseaux à Đux continu, de nature logistique qui pourvoient par exemple aux besoins d'une guérilla où les mécanismes priment souvent sur la volonté politique, et enfin des réseaux "à rétention" de violence, qui autorisent l'accumulation d'armes et sont donc potentiellement déstabilisateur dans la mesure où les acteurs étatiques qui les animent peuvent par ce moyen maîtriser le calendrier de l'usage de la violence. Il nous reste à envisager les évolutions possibles de ces réseaux de violence dans le contexte mouvant actuel. Deux schémas sont possibles : le premier reposant sur la prolongation des tendances lourdes et interprétant les perspectives de contrôle de la politique de vente d'armes dont Stéphanie Neuman nous dit qu'elles n'ont jamais été aussi favorables. Le second supposant une modification profonde de la place des réseaux. L'optimisme raisonné de S. Neuman repose sur une analyse des bouleversements actuels. Avant ces transformations, la structure bipolaire des relations internationales assurait une certaine marge de manoeuvre aux pays acheteurs qui avaient la possibilité de faire jouer la concurrence idéologique entre les deux blocs, ou tout simplement la concurrence économique entre des producteurs d'armes dont le nombre augmentait. La situation actuelle est toute différente. La guerre du Golfe et l'implosion de l'URSS ont renforcé la primauté des USA sur le marché des armes, et seuls les producteurs les mieux enracinés, ceux bénéficiant d'un marché intérieur large, peuvent maintenir leurs positions; les Européens sont affaiblis et les pays producteurs du Sud risquent de connaître une véritable débâcle renforcée par le fait que, côté client, l'effet Golfe déplace la demande vers le matériel "haut de gamme" que, justement, les pays du Sud ne produisent pas. à ces changements techniques du marché qui placent les vendeurs, et surtout le plus puissant d'entre-eux, en position de supériorité structurelle s'ajoute le sentiment que la moralisation du commerce des armes est devenue nécessaire devant les dangers de la prolifération des armes nucléaires ou balistiques. Cette évolution psychologique de l'opinion permet aux USA d'imposer à leurs concurrents une "déontologie" des ventes d'armes qui ne peut que renforcer leur prééminence ${ }^{5}$. Dans ce contexte la marge de manoeuvre des pays acheteurs est rognée et $\mathrm{S}$. Neuman peut conclure avec un optimisme raisonné qu'un contrôle des ventes d'armes dans un marché dominé par un grand producteur quitte le domaine de l'utopie pour entrer dans celui des possibilités. La deuxième évolution possible repose sur la crise du secteur armements marquée par des oppositions fortes entre dimension économique et dimension politique qui entraînent un renforcement des contradictions d'intérêts à l'intérieur des bureaucraties et la montée en puissance des lobbies. Si on y ajoute le délitement ou l'éclatement de certains états (Chine, Yougoslavie) et les effets de ce qu'on pourrait appeler le syndrome soviétique - c'est-àdire une politique de dumping sauvage d'armes classiques ${ }^{6}$ sur laquelle se greffe une dissémination de matériels, de techniques et de cerveaux, dans le secteur balistique ou nucléaire -, on se trouve en face d'une situation internationale qui rend flou le lien entre les effets généraux et les tactiques particulières : chacun gérant au plus prêt ses 
intérêts, sans se préoccuper des conséquences globales. Dans cette hypothèse, mesurer les effets sur les relations internationales des réseaux internationaux de violence consiste moins à dessiner les contours d'une géopolitique grandiose qu'à mettre en évidence une logique de bricolage appuyée sur la multiplication des réseaux de toute nature au service d'acteurs plus nombreux et, pas du tout monolithiques quand on a bien en tête les contradictions internes des états et la concurrence acharnée qui les oppose. Dans cette vision les pôles stables que disent être les états occidentaux jouent largement dans l'analyse le rôle de leurre ${ }^{7}$, et le schéma rassurant d'un contrôle de la prolifération des armes qui tiendrait compte, à la fois des intérêts généraux et des intérêts particuliers et contradictoires des blocs, ou des puissances, sera probablement remplacée par une dérégulation globale, terreau fécond pour la prolifération de la violence ${ }^{8}$.

\section{NOTES}

1. Table ronde organisée par Stephanie Neuman, dont les articles de Keith Krause, R. Bates Gill, Frederic S. Pearson, Didier Bigo sont issus. Les textes publiés dans ce numéro de Cultures \& Conflits seront repris ultérieurement par une revue américaine afin d'amplifier l'écho de la discussion que nous avons entamée avec le numéro sur "Mafia, drogue et politique" et que nous poursuivons avec celui-ci.

2. Voir l'article de Michel Wieviorka, "Le réseau de la terreur : une hypothèse à revisiter.

3. voir l'article de Didier Bigo, "Les attentats de 1986 en France : un cas de violence transnationale et ses implications". Cet exemple pourrait facilement être complété par d'autres comme le montre l'imbroglio-politico-judiciaire à propos de l'implication de la Libye dans les attentats contre la Panam et UTA.

4. Pour une analyse détaillée des rapports entre mafia, drogue et politique, et de la frontière qui les sépare, voir le $n^{\circ} 3$ de Cultures \& Conflits.

5. Notamment par le monopole de la catégorisation des pays en conflits entre "agresseur" et "stabilisateur" dont disposent les USA après la guerre du Golfe. Ils utilisent un mécanisme rodé par la publication annuelle de la liste des états terroristes du département d'état.

6. Bien que leur image ait pâli pendant la guerre du Golfe, certains des matériels soviétiques restent très attractifs (Mig 29 par exemple).

7. Sur la faiblesse des grandes puissances à peser sur les conflits dans le nouvel environnement international de concurrences imparfaites, voir Etudes polémologiques, $\mathrm{n}^{\circ} 40$, : transferts d'armements et conflits locaux, et Cultures \& Contlits $\mathrm{n}^{\circ} 1:$ la prolongation des conflits.

8. Sur cette question de da difficulté du contrôle de la prolifération, cf. B. Warusfel, Cultures \& Conflits, $\mathrm{n}^{\circ} 2$ et K. Krause, "Transferts d'armements et gestion des conflits le cas de la guerre Iran/Irak" dans ce numéro. 


\section{RÉSUMÉS}

Réseaux internationaux de violence, l'expression peut paraître obscure mais elle est la seule à rendre compte de la violence des attentats dits terroristes, de celle engendrée par des trafics aussi divers que celui de la drogue, des explosifs ou des armements lourds, ainsi que de l'activité des administrations qui parfois les animent ou de la politique de certains états. Le champ ainsi balisé concerne autant les relations interétatiques que celles existant entre états, guérillas, réseaux mafieux ou organisations clandestines c'est-à-dire entre "acteurs souverains" et acteurs "libres de souveraineté" pour reprendre le vocabulaire de J. N. Rosenau.

It is necessary to analyse the international networks of violence in order to understand how, sometimes, besides or within states relationships, the autonomisation of some bureaucracies or private interests appears, giving to any " intermediary " a real power within arms transfers. We must also avoid a too statocentric vision and raise the question of the place of the transnational actors in relation to the irruption of widespread violence.

\section{INDEX}

Mots-clés : sociologie des conflits (polémologie), réseaux transnationaux, violence

\section{AUTEUR}

\section{DANIEL HERMANT}

Professeur agrégé d'histoire, directeur de publication de Cultures \& Conflits. 